\title{
Escuelas Normales Rurales en México: movimiento estudiantil y guerrilla
}

\section{Rural teachers' schools in México: student movement and subversion}

\author{
Yessenia Flores Méndez \\ El Colegio de Tamaulipas, Ciudad Victoria, México \\ yessenia.flores@tam.gob.mx \\ ORCID: https://orcid.org/oooo-oooI-8878-9437
}

ISSN-OI85-4259; e- ISSN: 2007-9I76

DoI: http://dx.doi.org/I0.28928/ri/872019/aot3/floresmendezy

\begin{abstract}
Resumen
El objetivo de este artículo es exponer las causas de la participación de las Escuelas Normales Rurales (ENR) en el movimiento estudiantil mexicano de los años sesenta que culminó en 1968, así como los efectos posteriores, entre ellos, la aplicación de la reforma a la educación rural de 1969 que eliminó I5 de 29 ENR, y las transformó en Escuelas Secundarias Técnicas Agropecuarias (ETA). El gobierno y la Secretaría de Educación Pública (SEP) argumentaron la reorganización de la educación rural mediante la separación entre el ciclo secundario y el profesional (normal) para profesionalizar la carrera de maestro y la creación de un nuevo subsistema de educación media superior. Los dirigentes estudiantiles y alumnos normalistas rurales se opusieron a dicha reforma, $y$ a pesar de las protestas no pudieron detener su ejecución. Este trabajo tiene un enfoque de historia social, desde abajo. También retomamos los aportes de James Scott, quien propone interpretar la conducta política de los grupos subordinados mediante las estrategias de resistencia. La investigación está documentada con expedientes de la versión pública de la Secretaría de Gobernación.

Palabras clave‡ movimiento social, resistencia, reforma educativa, educación rural, guerrilla.
\end{abstract}

\begin{abstract}
The objective of this article is to explain the causes of the participation of the student leaders of the Rural Normal Schools (ENR) in the Mexican student movement of the sixties that ended in 1968. As well as the subsequent effects, among them, the application of the educational reform of 1969 that eliminated fifteen of twenty-nine Rural Normales. The transformation of half of ENR in Secondary Technical Agricultural Schools (ETA). The government and the SEP argued the reorganization of rural education, by separating the secondary cycle of the professional cycle (normal) to professionalize the teaching career and with the creation of a new subsystem of upper secondary education. But the student leaders and rural normalista students rejected the reform to normal education, in spite of the protests they could not stop its implementation. This work has a social history approach, from below. We also return to the contributions of James Scott, who proposes to interpret the political behavior of subordinate groups through strategies of resistance. The investigation is documented with files of the public version of the Ministry of the Interior.
\end{abstract}

Keywords: Social movement, Resistance, Educational reform, Rural education, Guerrilla.

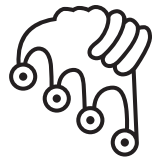

IZTAPALAPA

Agua sobre lajas 


\section{Introducción}

ste trabajo converge entre la historia de la educación y la historia de los
movimientos sociales. Los estudios sobre la educación rural y en particular
sobre las escuelas normales rurales se han realizado desde diferentes ópticas: las reformas, prácticas educativas y el movimiento estudiantil. Las Escuelas Normales Rurales (ENR) creadas en los años veinte en México fueron producto de la Revolución de I9IO, movimiento en el que los campesinos exigieron justicia social y demandaron tierras. En el periodo posrevolucionario, la educación rural fue un proyecto que perseguía mejorar las condiciones de la población del campo. En ese plan las ENR tuvieron un papel importante. Durante el cardenismo (19341940) recibieron impulso al crecer el número de escuelas y ser refuncionalizadas en Escuelas Regionales Campesinas (ERC) que unieron la enseñanza agropecuaria y la normalista. En este periodo se aplicó la educación socialista y los planes de estudios incluyeron orientación socialista y materialismo histórico.

En la década de los cuarenta el gobierno de Manuel Ávila Camacho impulsó la política económica de industrialización del país. La política educativa fue readecuada y la educación rural dejó de considerarse prioritaria. Las ENR en esta etapa quedaron desvinculadas por la falta de un plan para las zonas rurales (Greaves, 2010:197). Se anuló la escuela socialista, pero la ideología permaneció en las escuelas cuya matrícula escolar provenía de zonas rurales e indígenas. Las 35 ERC fueron separadas en normales rurales y escuelas prácticas de agricultura (EPA). Las 20 ENR subsistentes se reorganizaron en Io de hombres y Io de mujeres. En los años cincuenta las EPA fracasaron y se revirtieron en ENR, sumando 29 planteles y aumentaron su población estudiantil. ${ }^{1}$ No obstante, en 1969 sufrieron otro cambio radical y sobrevino su participación en el movimiento estudiantil que las redujo a I4.

1 Las erc de Roque, Guanajuato; La Huerta, Michoacán; Aguilera, Durango; El Quinto, Sonora; Mactumactzá, Chiapas y Champusco, Puebla, en I94I fueron convertidas en EPA y en 1959 reorganizadas en ENR. Otras ENR se fundaron en los años cincuenta: 
La historiografía del movimiento estudiantil mexicano ha destacado el papel de los estudiantes universitarios, pero no de los normalistas rurales. Los estudios previos también se han centrado en el movimiento de 1968 en la capital y no en la provincia. Sin embargo, este movimiento tuvo carácter nacional, porque en la lucha participaron en diversos grados estudiantes de distintos centros educativos del país. Las normales rurales distribuidas en la república mexicana parecían dispersas pero tuvieron un papel importante. Los actores principales fueron jóvenes capitalinos y la intervención de los de provincia tuvo diversa intensidad, fue fuerte en unos estados y débil en otros (Guevara, 1988: 47). Tratamos de posicionar la participación de las normales rurales y de los estudiantes en este movimiento social, así como descentrar la mirada hacia las entidades federativas.

Trabajos recientes han dado una mirada a las normales rurales en el movimiento estudiantil de 1968. Evangelina Terán, al referirse a la trascendencia de este movimiento, señala que "los normalistas rurales participaron, y que la lucha estudiantil se basó más en demandas políticas que académicas” (Terán, 2009). Sergio Ortiz menciona que la movilización de las normales rurales también planteaba demandas académicas con respecto a las reformas educativas. "La reforma educativa de 1969 se justificó con argumentos pedagógicos, pero fue una medida para someter al normalismo rural que en 1968 jugó un papel protagonista como foco del proselitismo de izquierda y con influencia del Partido Comunista Mexicano" (Ortiz, 20II: 387).

Marcelo Hernández afirma que en la reforma educativa de 1969, "el argumento fue la profesionalización de la carrera de maestro. Pero también fue efecto del movimiento estudiantil de las normales rurales; el gobierno solucionó el conflicto con la reorganización que consistió en separar el ciclo secundario del ciclo normal" (Hernández, 2015: 240).

Aleida García, por su parte, estudió la politización y participación solidaria de los estudiantes y maestros normalistas rurales que apoyaron el movimiento campesino y guerrillero en Chihuahua. Afirma que los orígenes de clase y las tradiciones pedagógicas (educación socialista) explican la adhesión de los estudiantes de las ENR a los movimientos (García, 2015).

Alicia Civera sostiene que los normalistas rurales, guiados por sus maestros, simpatizaron con el Partido Comunista Mexicano, apoyaron movimientos sociales, como el magisterial en los cincuenta y el estudiantil en los sesenta, y todo ello culminó con el cierre de un número importante de ENR. Este momento representó la segunda crisis de estas escuelas, después de la de 1940, que expresó la paraliza-

Zaragoza, Puebla; Santa Teresa, Coahuila (1959); y Atequiza, Jalisco (1950). 
ción a partir de dos posturas: por una parte, quienes intentaron independizarse del gobierno bajo el PCM y de una política izquierdista influida por la Revolución cubana que llevaría a algunos normalistas a sumarse a la guerrilla, y por otra parte quienes aceptaron sumarse a las redes del Estado autoritario. Fueron dos vías para exigir sus derechos y mejorar las condiciones de los pobres. Por ello no es posible comprender a los normalistas si no se considera su vulnerabilidad y su ubicación en las estructuras de poder (Civera, 2013).

La argumentación teórica y metodológica de este trabajo se sustenta en una historia social que se centra en los sectores olvidados y marginados. Retomamos los aportes de James Scott, quien propone enfocar no las formas aparentes de la hegemonía, sino las prácticas cotidianas, y no privilegiar los discursos oficiales sino los códigos ocultos, para descifrar las relaciones de poder entre dominados y dominadores. Propone interpretar la conducta política de los grupos subordinados mediante las estrategias de resistencia que utilizan. Las relaciones de poder son relaciones de resistencia. Los dominadores utilizan la estigmatización; en este caso, las autoridades educativas lanzaron una campaña para desprestigiar a los estudiantes normalistas rurales por su rebeldía y desobediencia, y ejercieron presión mediante sanciones. También diseñaron una estrategia, mediante la reforma educativa para buscar una respuesta a las problemáticas existentes en el sistema normalista rural. Las técnicas que usan los subordinados en el discurso público son las protestas, huelgas y peticiones (Scott, 2000: 8I). La categoría de resistencia ayuda a entender la relación de los normalistas con el poder.

En su larga trayectoria histórica, las normales rurales han sido reformadas. En I968 fueron reprimidas, pero sus conflictos no terminaron ahí, sino que se avivaron y continuaron en los setenta y ochenta. Entre 2003 y 2005 El Mexe tuvo un conflicto y la cerraron, y un movimiento posterior logró su reapertura sin internado. Ayotzinapa fue agredida en 2014 con la desaparición de 43 estudiantes, y las protestas siguen por aumentar la matrícula y las becas en Cañada, San Marcos, Tiripetío y Panotla. Mi interés por estudiar las normales en el 68 parte de mi preocupación por comprender $y$ aportar desde una perspectiva histórica y social, para conocer el pasado, entender el presente y replantear el futuro de dichas instituciones formadoras de docentes. A cinco décadas del movimiento estudiantil de 1968 es preciso hacer un análisis, dar algunas aportaciones y reflexionar sobre este suceso. Es claro que las normales rurales se resisten a desaparecer; defienden la existencia de este modelo de educación rural.

En este panorama es preciso responder a las siguientes preguntas: ¿Cuál fue la participación de los normalistas rurales en el movimiento estudiantil de 1968? ¿Por 
qué cerraron la mitad de normales rurales en 1969? ¿Qué factores propiciaron el cierre y refuncionalización de las ENR en ETA? ¿Cuál fue el impacto de la reforma educativa de 1969? ¿Cómo reaccionaron y resistieron los estudiantes ante la reforma educativa?

La estructura del texto responde a tres apartados: el primero contiene algunos antecedentes de la participación de los normalistas rurales en el movimiento estudiantil mexicano. El segundo trata del papel que desempeñaron las Normales Rurales en 1968. El tercero abarca el proceso de implementación de la reforma drástica en las normales rurales y la resistencia de los estudiantes, hecho que no se olvida.

\section{El movimiento estudiantil normalista rural en los años sesenta}

Desde 1935, época de la educación socialista, las normales rurales se agruparon en la Federación de Estudiantes Campesinos Socialistas de México (feCSM). Dicha organización militaba en la Confederación de Jóvenes Mexicanos (СЈM), sector juvenil del partido oficial desde 1939. Después de 1956 en varias normales rurales creció la inconformidad frente a la pasividad con que la сJM abordaba los problemas de esas escuelas. Entre 1960 y 1964, las normales rurales pasaron por una división debida a la disputa por la representación. Desde 196I se conformaron dos grupos, el primero que apoyaba a Lucio Cabañas de Ayotzinapa, Guerrero, y el segundo a Antonio Valtierra, de Salaices, Chihuahua. La división se dio en el Congreso de El Mexe cuando Cabañas salió electo secretario de la FECSM, y el grupo perdedor formó el Consejo Nacional Permanente (CNP) de ENR. ${ }^{2}$

Mónica N. López sostiene que la escisión de las enr se dio porque Antonio Valtierra era el candidato de los dirigentes de la Juventud Comunista (Jc) del Partido Comunista Mexicano (PCM). Pero la elección fue controlada por la facción de la сјм y su dirigente, Liderato Montenegro, que favorecieron a Cabañas, quien no

2 La fecsm con Cabañas representaba a I5 normales, Tiripetío y La Huerta, Michoacán; Ayotzinapa, Guerrero; Teteles, Champusco y Zaragoza, Puebla; Tamazulapan y Reyes Mantecón, Oaxaca; Mactumactzá, Chiapas; Galeana, Nuevo León; Tamatán, Tamaulipas; Takak, Yucatán; Hecelchakan, Campeche; El Quinto, Sonora y Perote Veracruz. Por otro lado, el cnp liderado por Zenón Ramírez controlaba II planteles: Salaices y Saucillo, Chihuahua; Aguilera, Durango; Roque, Guanajuato; Panotla, Tlaxcala; Jalisquillo, Nayarit; San Marcos, Zacatecas; Palmira, Morelos; Santa Teresa, Coahuila; El Mexe, Hidalgo y Cañada Honda, Aguascalientes. AGN-SG, Caja 6I, Legajo I/3I, Foja I8, 26 de marzo de 1963. 
cuestionó la militancia de la FECSM en la CJM. El PCM influyó en la separación de algunas ENR de la FECSM y en la creación del CNP, porque continuaban reconociendo a la dirigencia de la сјм, vinculada al partido oficial (López, 20I6: II9).

A finales de 1962, en el Congreso de la FECSM en El Mexe, Hidalgo, para elegir nueva dirección, I7 escuelas normales rurales estaban de acuerdo, mientras que nueve la desconocieron. En el xviri Congreso en la enr de Galeana, Nuevo León, en mayo de 1963, el objetivo era buscar la unidad de las normales. Pero no hubo representación, solo asistieron $\mathrm{I} 6$ delegados, algunos abandonaron la FECSM, y se unieron al Consejo Nacional Permanente con sede en Roque, Guanajuato. Desde abril, los disconformes se habían reunido en Salaices, y acordaron no asistir al congreso. ${ }^{3}$

En el intento separatista que promovieron las organizaciones estudiantiles de la oficial сJм, los dirigentes de esta agrupación llamaron a la reunificación de las ENR en el Congreso de la fecsm en Galeana, "donde nombraron una dirección común y lograron retener solo un tiempo más en la CJM al sector mayoritario de ENR" a excepción de las normales del $\mathrm{CNP}^{4}{ }^{4}$

Cuevas afirma que tras la división de la слм en dos facciones se conformaría una central estudiantil independiente, desligada del gobierno y del partido oficial. Con la fragmentación de la FECSM, las ENR del CNP se sumaron al proyecto de construcción de la Central Nacional de Estudiantes Democráticos (CNeD), (Cuevas, 1984: 157). El pсм realizó en Morelia, del I5 al I7 de mayo de I963, la I Conferencia de la CNED, en la cual fue emitida la Declaración de Morelia. Ahí participaron las EnR del CNP. A la II Conferencia de la CNED acudieron representantes del CNP integrado por IO enr de El Mexe, Hidalgo; Aguilera, Durango; Palmira, Morelos; Panotla, y Xocoyucán, Tlaxcala; Cañada Honda, Aguascalientes; Saucillo y Salaices, Chihuahua; San Marcos, Zacatecas y Santa Teresa, Coahuila. ${ }^{5}$

A finales de 1964, la feCSM con Heliodoro Ramos Venegas ratificó la unidad de las ENR en la FECSM, pero continuó sin desprenderse plenamente de la CJM. Sin embargo, advirtió en una reunión en El Mexe que la facción comunista organizaba el Congreso de Hecelchakán y pretendían apoderarse de la FECSM o dividirla. El resultado fue la expulsión de líderes estudiantiles comunistas de la fecsm. En dicho congreso transitaron hacia una nueva dirigencia que abrazó finalmente una

AGN-SG, Caja 6I, Legajo I/3I, Foja 32, I de mayo de I963.

4 Boletín I, Comité de Orientación Ideológica, Enr Reyes Mantecón, Oaxaca, 28 de agosto de 1963.

5 AGN-SG, DFS, FeCSM, Caja 6I, Legajo I/3I, Foja 174, 7 de mayo de 1964. 
militancia en el movimiento estudiantil democrático que encabezaba la Juventud Comunista (López, 2016: 18I).

La tendencia de algunas normales rurales de aproximarse a la CNED fue impulsada por varios sucesos. A fines de 1964, el gobernador de Chihuahua, Práxedes Giner declaró que los internados de las normales rurales eran guaridas de comunistas, y encarceló a estudiantes. Así "la Juventud Comunista del PCM se apropió de la FECSM, votando la mayoría de los dirigentes estudiantiles durante el Congreso de Hecelchakán en mayo de 1965 para ingresar a la CNED" (Cuevas, 1984: 85).

Entre las causas que llevaron a los dirigentes estudiantiles a simpatizar con la izquierda fueron varias. En los años cincuenta surgió un movimiento agrario encabezado por Rubén Jaramillo en Morelos, que protestó por la imposición del partido oficial y la violencia caciquil. En 1962 fue eliminado, y el terreno se abonó para que se gestaran las guerrillas. Fue un hecho que marcó a la izquierda e inspiraría a los jóvenes activistas (Castellanos, 2007).

El 23 de septiembre de 1965, un grupo de estudiantes, maestros normalistas y campesinos crearon el primer foco guerrillero, inspirado en el manual de guerra de guerrillas del Che Guevara y en el asalto al cuartel de Moncada, Cuba, por Fidel Castro el 23 de julio de 1953. Hicieron el asalto relámpago en un cuartel militar de la población rural de Madera, Chihuahua. Liderados por Pablo Gómez, médico y profesor normalista, y Arturo Gámiz un joven maestro que denunció la situación de los campesinos y participó en la guerrilla rural. Murieron en el intento, pero ese hecho inspiró a posteriores organizaciones chihuahuenses, así como a la mayor guerrilla urbana mexicana que nombró a la Liga Comunista 23 de Septiembre en honor a esa fecha. El ataque fallido produjo una onda expansiva que transcendería para convertirse en símbolo de lucha armada de otros jóvenes del país (Castellanos, 2007).

El germen guerrillero fue atribuido a la ENR de Salaices, y creyeron que se expandiría por todas las del país. Los estudiantes, indignados, exigieron peticiones económicas y educativas. Para octubre de 1965, los líderes estudiantiles se reunieron en la Normal de Saucillo, donde repudiaron a Giner por su política anticampesina, causante de los sucesos de Madera," "participando más activamente en la integración de la CNED" (Cuevas, 1984: 85). Los dirigentes de las ENR decidieron tomar acción en las movilizaciones que la Juventud Comunista venía convocando desde I963.

A finales de los cincuenta y principios de los sesenta, mientras obreros y magisterio eran reprimidos, los campesinos de Morelos, Sonora, Nayarit, Baja California y Chihuahua se lanzaron a una lucha por las tierras. En Chihuahua, como en otros estados del país, la pugna agraria había cobrado impulso con el apoyo del alumnado y el magisterio de las ENR. La modalidad de activismo colectivo más recurrente en 
Chihuahua fue la toma de tierras, y en 1963 proliferó con la participación de la Unión General de Obreros y Campesinos de México (Ugocm), otros partidos políticos y escuelas normales que invadían predios latifundistas. Campesinos y estudiantes irrumpían. En la enR de Saucillo los normalistas encabezaban las acciones. Las ENR eran espacios en que había estudiantes muy politizados que hacían circular literatura comunista. Otras organizaciones ya se habían relacionado con estudiantes y maestros rurales de Sonora, Durango, Guanajuato, Guerrero, Estado de México, Morelos, Chiapas, Yucatán y Chihuahua. En los estados del sur apenas se gestaba el proceso de insurrección (Castellanos, 2007).

En Guerrero los estudiantes de Ayotzinapa iniciaron un movimiento ante la amenaza del gobierno local de clausurar esa normal. Cuando Lucio era dirigente de la ENR, convocaba a los estudiantes a la huelga universitaria y a otros movimientos sociales. A Cabañas lo invitaron a sumarse al movimiento de la Liga 23 de Septiembre, pero en ese momento, como militante de las Juventudes Comunistas y líder de las ENR, lo consideró radical.

En Guerrero la guerrilla rural tuvo un desarrollo más amplio que en otras entidades. La actividad armada de Lucio Cabañas y Genaro Vázquez se debió al cacicazgo local, los fraudes electorales y la miseria campesina. Genaro tomó las armas por la violencia institucional a la que fue sometida su organización y también como resultado del contexto internacional (Barrera y Sarmiento, 2008: 657). A partir de 1967, luego de la masacre de Atoyac, Lucio decidió cristalizar la idea de organizar un grupo armado y lanzarse a la lucha guerrillera, pues consideró que los canales institucionales se habían cerrado. En su etapa de estudiante y durante su estancia en la escuela de cuadros del PCM había conocido las experiencias guerrilleras que desde finales de los cincuenta prosperaban en América Latina. La lucha de Genaro era nacionalista y el objetivo de Lucio era instalar un régimen socialista.

En 1965 las ENR se declararon en huelga con la exigencia de que se mejoraran los servicios asistenciales, materiales, de enseñanza y de infraestructura. La SEP respondía parcialmente a las peticiones y empezó a elaborar una estrategia para solucionar la problemática de las normales. Desde 1963 se conocía la amenaza de cerrarlas y entregarlas a los gobiernos estatales, es decir, que la federación dejaría de otorgarles financiamiento. ${ }^{6}$

Hacia noviembre de 1965 hubo un encuentro entre el Comité Central de lucha de la CNed y el comité de la fecsm en la normal de Panotla, Tlaxcala. Acordaron

6 AGN-SG, DFS, FECSM, Caja 6I, Legajo I/3I, 3 de octubre de 1963. 
la unidad para el movimiento nacional (Peláez, 1980). Las ENR se unieron en la FECSM, en el Congreso Constituyente de la CNED en abril de $1966 .{ }^{7}$ Mario Aguilera Dorantes, Oficial Mayor de la SEP, impidió desde 1963 el posible acercamiento entre la FECSM y el CNP-ENR. Las acciones emprendidas antes de la sucesión presidencial de 1964 en contra de las ENR del norte del país, condujeron a Aguilera a considerar necesaria la unidad, aunque existiera el peligro de que la facción comunista que dirigía al CNP-ENR llegara a dominar la FECSM unificada (López, 2016ः 160).

El año de 1966 fue clave en el desarrollo del movimiento estudiantil mexicano. Lo acontecido en Morelia fue un antecedente de lo que transcurriría dos años después en la Ciudad de México. Las normales de Tiripetío y La Huerta, Michoacán, se sumaron (Zermeño, 1978 y Guevara, 1988). Como parte de las acciones del gobierno autoritario, los dirigentes del PCM y de la CNED sufrieron persecución y encarcelamiento; los estudiantes universitarios y normalistas rurales reclamaron la liberación de los presos políticos.

La fecsm, presidida por Antonio Torres Valle, apoyó a los estudiantes de Morelia en el paro nacional que convocaron para secundarlo en las normales rurales. Solo respondieron las enr de Atequiza, Jalisco; Salaices, Chihuahua y Aguilera, Durango. Consideraron que todo paro entre los estudiantes de las normales calendario A no daría resultados por estar en periodo de exámenes finales, pero sí en las de calendario $B$ donde podía prosperar algún movimiento. Enviaron propaganda de la CNED a todas las normales rurales mientras preparaban su pliego petitorio para iniciar la agitación en todo el sistema de educación normal rural en enero de $1967{ }^{8}$

En el xxi Congreso de San Diego Tekax, Yucatán, de I967, el comité encabezado por Matías Rodríguez confirmó la militancia oficial de la FECSM en la CNED, al ocupar este la secretaría de Acción Campesina de dicha central de estudiantes democráticos. Con la unidad de las ENR, la JC logró controlar todas las sociedades de estudiantes. La militancia de la FECSM en la CNED cambió el discurso político y, en consecuencia, la relación con las autoridades educativas del gobierno de Gustavo Díaz Ordaz.

A principios de 1967, la CNED organizó la "Jornada por la Democratización de la Educación Superior" e invitó a los líderes normalistas. Las ENR venían haciendo su propio movimiento y manifestando sus demandas; mientras tanto, el gobierno, la SEP y la Dirección General de Educación Normal (DGEN) estaban preparando

7 AGN-SG, IPS, abril, I966.

8 AGN-SG, IPS, SEDENA, I5 octubre, 1966. Situación que prevalece entre el estudiantado de las escuelas normales. 
algunos cambios. A mediados de 1967 realizaron la "Asamblea Nacional de Educación Normal", que propuso una reforma educativa para reestructurar las normales rurales. Proponían orientar la preparación vocacional de los jóvenes del campo y encaminarlos hacia una formación técnica y no ver la carrera de maestro rural como única opción de estudios. Las autoridades argumentaron que existía sobredemanda estudiantil. Planearon suprimir o transformar el ciclo secundario y crear secundarias rurales técnico-agrícolas, y sustituir las ENR por Centros de Educación Normal Rural. ${ }^{9}$

El secretario de la SEP, Agustín Yánez, planteó ofrecer alternativas a los alumnos que egresaran del ciclo secundario de ENR para seguir otras carreras. ${ }^{10}$ Propuso que ingresaran en otras instituciones, al Instituto Politécnico Nacional y a la Escuela Nacional de Agricultura. La Sep planeaba eliminar los internados, otorgar becas en efectivo y agregar un año al ciclo profesional (normal), medidas para terminar con los problemas estudiantiles y las huelgas. Estos anuncios provocaron la desconfianza de los estudiantes normalistas rurales.

Mientras tanto, la Juventud Comunista había promovido una campaña de reclutamiento de jóvenes militantes entre agosto y octubre de 1967 en universidades y normales (Cuevas, 1984:87). El año de 1968 inició con una apretada cadena de luchas estudiantiles. La CNED se recuperó de la detención de líderes en 1966 y amplió su influencia con presencia en las ENR. El 3 de febrero de 1968 fue bloqueada la "Marcha por la Libertad" a la que se había convocado y que iría de Guanajuato a Morelia. La CNED buscaba reactivar sus acciones en provincia. En mayo realizó un segundo congreso en la Ciudad de México y convocó nuevamente a la movilización (Tecla, 1994: 29). A principios de ese año el enfrentamiento de las normales rurales con las autoridades educativas se reavivó y representantes de las ENR pasaron a ocupar el Consejo de Vigilancia de la CNED a partir del congreso de mayo (Cuevas, 1984: II6). Las normales rurales se habían convertido en los peones de lucha más fieles de la CNED, pues "desde 1965 constituían la columna vertebral de dicha organización", al aportar recursos para las movilizaciones. ${ }^{11}$

Antes de que estallara el movimiento estudiantil de julio-octubre de 1968, los normalistas rurales ya estaban movilizados. Las ENR, mediante la FECSM y convocados

9 AGN-SG, DFS, FeCSM, Legajo 2-3I, Caja 62, I de julio de I967.

10 Pasajes del discurso del secretario de la SEP, Agustín Yáñez, al inaugurar la Asamblea Nacional de Educación Normal Rural, 3 de julio de I967. AGN-SG, DFS, FECSM, Caja 62, Legajo 2-3I.

11 AGN-SG, DFS, FECSM, Caja 6I, Legajo I/3I, I9 de abril de 1965. 
por la CNED, en febrero participaron en la Marcha de la Libertad, que desembocó a una huelga general por demandas económicas. Las autoridades cumplieron las peticiones y les dieron un ultimátum de no sumarse ni provocar agitaciones.

En mayo de ig68 se celebró el xxir Congreso de la Fecsm en la enr de Mactumactzá, Chiapas. Los normalistas expusieron algunas demandas educativas, denunciaron una crisis en el sistema educativo y en las ENR, defendieron una reforma a la educación normal y se pronunciaron a favor de una educación científica, democrática y popular, de acuerdo con sus estatutos. A esta reunión asistieron Rolando Waller de la CNED y Celso Garza de la JCM, lo cual demuestra la línea que siguió la fecsm. ${ }^{12}$ Pero las enr fueron investigadas; la Dirección Federal de Seguridad pidió informes de matrícula, directores y comunidades que rodeaban las escuelas. Hacia 1968 funcionaban 29 normales rurales, ocho de mujeres y 2I de hombres, con una matrícula cercana a los Io 000 estudiantes normalistas rurales, es decir, 7253 alumnos y 2434 alumnas, lo cual representaba un contingente numeroso. ${ }^{13}$

\section{La participación de las normales rurales en el movimiento del 68}

En 1968 los estudiantes se rebelaron desde los Estados Unidos y México, en Occidente, hasta Polonia y Checoslovaquia en el bloque socialista, estimulados por la erupción de mayo en París, epicentro de un levantamiento estudiantil continental (Hobsbawm, 1995: 305). Para Guevara Niebla, el movimiento estudiantil mexicano se gestó con varios sucesos: la ocupación del Politécnico Nacional en 1956, el movimiento magisterial (1958) y ferrocarrilero (1959), que crearon las condiciones para que surgiera la rebeldía de estudiantes que lucharon por la democratización del país. El movimiento desarrollado en los sesenta culminó en 1968 (Guevara, 1988: 57).

En el sexenio de Díaz Ordaz (1964-1970) sucedió el movimiento médico, la entrada del ejército en las universidades y la represión a campesinos. Según Zermeño, "el movimiento del 68 fue un episodio de lucha democrática, los estudiantes vivían un régimen donde las decisiones eran tomadas por el Estado, buscaban un cambio de sistema, una sociedad democrática y mayor libertad de participación" (Zermeño, 1978: 23).

12 AGN-SG, DFS, FECSM, Caja 62, Legajo, 4/3I, 4 de junio de 1968.

13 AGN-SG, DFs, FECSM, Caja 62, Legajo 4-3I, julio de I968. 
En la primera etapa del movimiento de 1968, la Federación de Estudiantes Técni$\cos$ (FNET) del IPN y la CNED organizaron marchas independientes que culminaron el 26 de julio. El Estado habló de una supuesta "conjura o conspiración comunista" que quería desestabilizar al gobierno mexicano (Del Castillo, 20I2). El I8 de julio fue descubierto un supuesto complot para derrocar al gobierno del país, al ser detenidos I4 jóvenes acusados de conspiradores.

La FNET llamó a una manifestación en protesta por la represión y ocupación de sus escuelas. La CNED y el Partido Comunista Mexicano (PCM) conmemoraron la Revolución cubana, hubo enfrentamientos entre estudiantes, policías y militares, que terminaron con violencia; el ejército irrumpió contra los supuestos agitadores comunistas. En la segunda etapa, el gobierno se replegó, el 8 de agosto se unieron la UNAM, IPN y Normales Rurales (Zermeño, 1978). El movimiento se extendió desde agosto y finalizó el 2 de octubre, con la masacre o matanza en Tlatelolco.

En septiembre de 1968, miembros del comité de la FECSM se trasladaron a varias ENR B, para tomar un acuerdo de apoyo al movimiento estudiantil. Los estudiantes seguidores del exsecretario Matías Rodríguez, que seguía ejerciendo control, fueron aconsejados de apoyar a nombre de la CNED. El secretario Rubén Rocha insistió en que debían hacerlo a nombre de las ENR. ${ }^{14 " H a b i ́ a ~ e s c u e l a s ~ q u e ~ n o ~ a c e p t a b a n ~ b r i n d a r ~}$ el apoyo que los podía afectar, pues la SEP los percibió y en caso de que se lanzaran a otro movimiento, procedería al cierre de algunos planteles y retiro de becas" ${ }^{15}$ En Mactumactzá, los estudiantes le indicaron a su delegado que no aceptara huelga en las ENR por el movimiento estudiantil, que solo se brindara apoyo moral.

La feCSM formó parte del Comité Coordinador de Huelga y luego del Comité Nacional de Huelga en agosto de 1968. Las demandas planteaban peticiones propias de las ENR, que solo participaron en el Consejo General de Huelga en solidaridad con el movimiento nacional (Peláez, 1980). Después de la represión estudiantil en la capital del país, el Estado se replegaría hacia las normales rurales.

El PCM y sus organismos juveniles como la Juventud Comunista Mexicana y la CNED, jugaron un papel importante en la coordinación del movimiento de 1966 y 1968. El PCM influyó pero no dirigió ni planeó (Zermeño, 1978: 23). Durante el movimiento del 68 se desarrolló una campaña anticomunista contra estas organizaciones (PCM, 1973: 237). También tuvieron un papel activo las pequeñas organizaciones de izquierda (Meyer, 2000).

14 AGN-SG, DFS, FECSM, Caja 62, Legajo 4/31, 1968-1974, 27 de septiembre de 1968.

15 AGN-SG, DFS, FECSM, Caja 62, Legajo 4/31, 1968-1974, 28 de septiembre de 1968. 
Según Aurelio Cuevas, durante los sucesos de 1968 en la capital del país solo un sector de las normales suspendió labores mientras que las demás fueron ocupadas por el ejército. Como las normales estaban regidas por dos calendarios A y B, su acción fue desorganizada al brindar apoyo al movimiento (Cuevas, 1984: II7).

A un mes del 2 de octubre, el 8 de noviembre las enr tipo B que estaban laborando eran Tamatán, Galeana, Hecelchakan, Jalisquillo, Saucillo, Salaices, Tekax y San Marcos; y en huelga, Atequiza, Ayotzinapa y El Quinto. Corrió la versión de un paro en apoyo al movimiento estudiantil de México, para protestar por la amenaza del secretario de la sep Agustín Yáñez, de la posible desaparición de las ENR. El día II pararon labores las ENR B por el cierre de las I4 ENR A que estaban en periodo vacacional. ${ }^{16}$

Las alumnas de la Normal Rural de Galeana, encabezadas por Herlinda Cantú, presidenta de la sociedad de alumnos, y estudiantes de la Juventud Comunista Mexicana (JCM) y otros universitarios visitaron la Universidad de Nuevo León para dar a conocer que "el alumnado de las normales rurales habían sido atacados impunemente por la SEP, que acordó clausurarlas por el único delito de haber apoyado el movimiento estudiantil del Distrito Federal". ${ }^{17}$

Los alumnos de las enr de Tamatán, Tamaulipas, repartieron volantes en las calles de Ciudad Victoria, informaron que: "I4 ENR fueron cobardemente clausuradas, fueron aprehendidos 8 compañeros de Jalisco, Nayarit; 13 de El Quinto, Sonora; 3 mujeres de Atequiza; uno de Perote, y represalias sufridas por la reacción del mal gobierno proimperialista de México, censura al estudiantado, por libertades políticas pisoteadas por los gorilas en el poder." ${ }^{18}$

La sociedad de alumnos "Emiliano Zapata" de la Normal de Jalisquillo, Nayarit (tipo B), se entrevistaron con el gobernador del estado a fin de que interviniera ante

16 AGN-SG, Circular de la CNED, 9 de noviembre, 1968. Las enR calendario A eran I3: Champusco, Teteles y Zaragoza, Puebla; Tenería, Edo. de México, El Mexe, Hidalgo, Tiripetío y La Huerta, Michoacán; Palmira, Morelos; Panotla, Tlaxcala; Mactumactzá, Chiapas; Perote, Veracruz; Reyes Mantecón y Tamazulapan, Oaxaca. Las enr B eran I4: Ayotzinapa, Gro; El Quinto, Sonora; Hecelchakan, Campeche; Jalisco, Nayarit; Aguilera Durango; Salaices y Saucillo, Chihuahua; San Diego Teyak, Yucatán; San Marcos, Zacatecas; Santa Teresa, Coahuila; Tamatán, Tamaulipas, Atequiza, Jalisco; Cañada Honda, Aguascalientes, y Galeana, Nuevo León.

${ }^{17}$ AGN-SG, IPS, Caja 1475B-05I, noviembre, 1968, problema estudiantil.

18 AGN-SG, DFS, FECSM, Caja 62, Legajo 4/3I, I968-1974, II de noviembre de 1968. 
las autoridades federales para evitar cerrar la escuela, que estaba como las ENR de Ayotzinapa y Cañada, rodeadas por el ejército, y el El Mexe y Panotla clausuradas. ${ }^{19}$

La CNED emitió una circular el i6 de noviembre, en la que expuso que el gobierno reaccionario, a través de la SEP, utilizando los viejos métodos, desde la amenaza, pasando por una serie de represalias contra los dirigentes de la FECSM, trataba de impedir la participación de los normalistas rurales junto a todo el estudiantado y pueblo de México en sus mejores causas, pero que habían fracasado, como habían fracasado las maniobras del ejército al rodear las escuelas para amenazar a los habitantes de las poblaciones circunvecinas en el afán de impedir que prestaran ayuda a los estudiantes del campo. Denunció públicamente la actitud chantajista de la SEP en el sentido de que habría de clausurar indefinidamente las I5 ENR calendario tipo A como represalia ante la actitud combativa de las escuelas tipo B que participaban decididamente en la lucha justa del estudiantado mexicano por las libertades democráticas.

Las normales B presionaron a las autoridades educativas para que no se clausuraran las normales A. El gobierno federal intentó cerrar las ENR tipo A, castigarlas por el apoyo de las ENR B al movimiento del 68. Los normalistas vieron la amenaza de la desaparición de todas las ENR, y su resistencia lograría la permanencia de la mitad de ellas. Ante las protestas y sin argumentos sólidos, el gobierno federal desocupó los planteles que intentaba suprimir y esperó al siguiente año, 1969, para terminar de diseñar y legitimar la reforma educativa que ya estaba anunciada $y$ preelaborada desde 1967 .

\section{Aplicación de la reforma educativa de 1969 y resistencia a la desintegración de las normales rurales}

Los líderes estudiantiles estaban preocupados por las reformas anunciadas en 1967. A principios de 1969, las autoridades notificaron la reforma a la educación superior para "democratizar la educación para la juventud". En febrero los normalistas realizaron el "I Seminario sobre la Reforma a la educación normal". Ahí surgió la Declaración de Atequiza, por cuyo conducto la FECSM se oponía a la reforma por su contenido político, que afectaba la educación popular.

La CNED se proclamó contra la aristocratización de la enseñanza y pidió solidaridad con las ENR. Manifestó "que México necesitaba más y mejores maestros, pero

19 AGN-SG, IPS, 9 de noviembre de 1968. 
la solución no era desintegrar el subsistema de educación normal rural, ni alargar la carrera, sino una transformación radical del contenido, orientación y métodos, que la colocara en posibilidad de servir a los cambios democráticos y revolucionarios que exigía el desarrollo del país" ${ }^{20}$

En abril de 1969, la SEP mediante la Dirección General de Educación Normal (DGEN) efectuó en Saltillo, Coahuila, el "iv Congreso Nacional de Educación Normal" con el que se oficializó la reforma educativa a las normales rurales, bajo el argumento pedagógico de la separación de los ciclos secundario y normal (plan de seis años) para profesionalizar la carrera de maestro (Barbosa, 1972: 270). En el Congreso de la fECSM celebrado en Ayotzinapa, en mayo, los dirigentes estudiantiles acordaron protestar por la reforma.

Del 28 de julio al 2 de agosto de 1969, en la Ciudad de México, la vin Asamblea Plenaria del Consejo Nacional Técnico de la Educación (Conalte) reafirmó aplicar los acuerdos de separar la secundaria y profesionalizar la enseñanza normal, decisión que propuso desintegrar las 29 ENR, y dejar 15 normales y I4 Escuelas Secundarias Técnicas Agropecuarias (ETA). Las normales rurales clausuradas y refuncionalizadas en ETA fueron I2: ocho de hombres, Jalisquillo, Nayarit; Santa Teresa, Coahuila; Tamatán, Tamaulipas, Xocoyucan, Tlaxcala; Reyes Mantecón, Oaxaca; Tekak, Yucatán; Zaragoza, Puebla y Salaices, Chihuahua. Y cuatro de mujeres, La Huerta, Michoacán; Champusco, Puebla; Palmira, Morelos y Galeana, Nuevo León. La reforma implicó el reacomodo del alumnado. ${ }^{21}$

Las autoridades expusieron la utilidad y el beneficio de la reorganización de la modalidad de educación rural, al crear un nuevo subsistema de educación media superior con las ETA. Expidieron el documento "Reformas fundamentales en el sistema de enseñanza Normal”, en el que ofrecieron aumentar las becas en las ENR exclusivas para jóvenes del campo y ampliar opciones de estudios superiores. Expidieron otro documento titulado "Más y mejor educación para los campesinos de México. Las Escuelas Tecnológicas Agropecuarias y las Escuelas Normales Rurales”. El discurso de la SEP argumentaba que no existían escuelas de nivel medio y superior en las áreas rurales. Esto sirvió de base para reorganizar las ENR y crear ETA. Estas

20

21

Con la nueva reestructuración del subsistema de Educación Normal Rural, las ENR que sobrevivieron fueron: Io de hombres, El Quinto, Son; Aguilera, Durango; San Marcos, Zacatecas; Atequiza, Jalisco; Tiripetío, Michoacán; El Mexe, Hidalgo, Tenería, Edo de México; Ayotzinapa, Guerrero; Mactumactzá, Chiapas; y Hecelchakan, Campeche. Y cinco de señoritas: Cañada, Honda, Aguascalientes, Panotla, Tlaxcala; Saucillo, Chihuahua; Teteles, Puebla; Tamazulapan, Oaxaca. 
secundarias capacitarían para mejorar las técnicas agropecuarias y la productividad rural. Es decir, no solo formarían maestros y maestras rurales, sino también técnicas y técnicos agropecuarios.

La SEP diseñó un organigrama al vapor en el que crearía nuevas instituciones educativas. La reforma educativa de 1969 no solo modificaba las ENR sino también reorganizaba todo el subsistema de educación rural. Los niños y las niñas del campo al salir de la primarias rurales podrían ingresar en las ETA (segunda enseñanza y capacitación para el trabajo rural en tres años), y después incorporarse en preparatorias técnicas, generales o agropecuarias, y posteriormente estudiar en normales, universidades, tecnológicos o escuelas superiores. Lo cual significaba mayor duración de estudios y costos.

Todavía quedan dudas y preguntas ¿Cómo decidieron cuáles escuelas permanecerían como normales y cuáles quedarían solo de secundarias? ¿Qué criterios determinaron la refuncionalización de ciertas normales? Probablemente permanecieron las normales que mantenían fuertes alianzas políticas. La reorganización no fue justa y la redistribución de planteles por regiones tampoco fue equitativa. Recordemos que gobernación estaba investigando la ubicación de las normales y que posiblemente eligieron cerrar las que no estaban rodeadas de muchas comunidades y población rural que provocaran una movilización campesina.

La SEP aplicó la reestructuración en el periodo de verano, cuando las ENR B estaban de vacaciones, y en las calendario A tuvo que desalojar al alumnado. Retiraron la alimentación de los internados para presionar y acataran las disposiciones. En algunas normales la transformación fue violenta; por ejemplo, en la de Palmira, de mujeres, reclasificada como secundaria, las alumnas denunciaron la entrada de granaderos en los dormitorios; se resistieron y llamaron a "todas a sus escuelas, no querían traidoras" ${ }^{22}$

La FECSM amenazó con apoderarse de los planteles en protesta por el nuevo plan educativo acordado por SEP, gobernación y gobernadores, que evitaron el apoderamiento. El gobierno utilizó a los campesinos para impedir que algunas ENR fueran tomadas por los alumnos; otras escuelas fueron ocupadas por la policía o el ejército. Los líderes normalistas acordaron tomar las ENR con apoyo de otras instituciones educativas.

Según Cuevas, en medio de las manifestaciones de los dirigentes normalistas entre julio y agosto, el ejército, la policía y miembros de la Confederación Nacional

22 AGN-SG, DFS, FECSM, Caja 63, Legajo 8/31, 2 de septiembre de 1969."La Normal de Palmira desaparece!", Cuernavaca, mimeografiado. 
Campesina (CNC) ocuparon las oficinas de la FECSM, ubicadas en El Mexe, Hidalgo, así como las normales más difíciles: La Huerta, Michoacán, Zaragoza, Puebla y Salaices, Chihuahua y hubo un intento de cooptar a los dirigentes de la FECSM, pero la ola de paros y disturbios continuó hasta septiembre en todas las normales (Cuevas, 1984).

Los líderes estudiantiles de las ENR fueron detenidos y encarcelados. El 8 de agosto de 1969, la FECSM emitió un comunicado conocido como el "Manifiesto de Ayotzinapa" en defensa de las ENR, contra la reforma antipopular y reaccionaria. Denunciaron el régimen de Díaz Ordaz, la represión militar y policiaca y la ocupación de las normales. La reforma fue calificada como impuesta y represiva, y reconocieron que era resultado de su participación en el movimiento del 68. Ante el fracaso de dividir y controlar la FECSM, el gobierno quitó escuelas. Los normalistas rechazaron la reforma y llamaron a luchar por cancelarla. No se oponían a la creación de ETA, aceptaron ampliar la educación rural y no solo mantener las ENR existentes sino abrir más escuelas de ese tipo. Estaban molestos y criticaron a las autoridades: ${ }^{23}$

¿Cuál era el objetivo que perseguía la SEP con sus reformas? Servir a los intereses de terratenientes, a la demanda de técnicos y mano de obra calificada, poner adelante las necesidades tecnológicas y omitir las clases campesinas. Someter el movimiento estudiantil porque las ENR eran centros de tradición revolucionaria, el maestro rural conocía las condiciones de vida del campesino.

Entre las declaraciones de los líderes detenidos, Miguel Zúniga, de la normal de Zaragoza, Puebla, secretario de conflictos de la FECSM, declaró que organizaban un movimiento para anular la reforma porque afectaba a los jóvenes campesinos, para quienes era difícil estudiar otra carrera de médico o ingeniero. En mayo, en el Congreso de Ayotzinapa discutieron sobre la reforma, que no fue bien vista por los estudiantes y acordaron revisarla antes de su aplicación, en el orden técnico y pedagógico y dar opinión a los alumnos. Desde los primeros meses de 1969 se habían reunido los representantes de 27 normales. Justificaron que "en el conflicto del 68 se fueron a la huelga en apoyo directo de sus propias demandas." ${ }^{24}$

La FECSM denunció la expulsión de 300 estudiantes de secundaria y normal por reorganizar las sociedades de alumnos. Las autoridades argumentaron que había que

23 AGN-SG, DFS, FECSM, Caja 63, legajo 9/31, foja 29I. En defensa de las normales rurales contra la reforma antipopular y reaccionaria, 8 de agosto de 1969.

24 AGN-SG, DFS, FECSM, Caja 62, Legajo 5/3I, I2 de agosto de 1969. 
castigar la rebeldía destructora para que las escuelas pudieran marchar en armonía. Los líderes pidieron la restitución y llamaron a parar esas acciones. Reprochaban la inactividad, falta de decisión y la clausura de internados.

La resistencia de los normalistas fue infructuosa, y fueron expulsados 500 alumnos, entre ellos 300 miembros de la Juventud Comunista, y destruidos todos los Comités de Orientación Política e Ideológica (COPI) que la CNED había fundado en las normales rurales. Concluyó así la actuación de las ENR y FECSM en la CNED, que con este duro golpe desapareció de las luchas estudiantiles del país (Cuevas, 1984).

El 3 de septiembre la FeCSM hizo un llamado a la solidaridad con la lucha de las normales rurales. Los 12 ooo estudiantes normalistas se lanzaron a la huelga general por la no desintegración de las ENR. Llamaron a no entrar a clases. Casual, la embestida contra las ENR se realizaba en un momento que trataban de impedir toda acción de estudiantes, a unos meses de la separación de las escuelas prevocacionales del IPN, y subir las altas cuotas en universidades de provincia.

El 23 de septiembre los líderes de la FECSM manifestaron una contrarreforma, pero no recibieron el apoyo esperado de los estudiantes normalistas rurales y de los universitarios, porque el movimiento estudiantil estaba reprimido. No recibieron apoyo de la unam y del ipn, solo de la Normal Superior. Solamente las EnR de Panotla y Ayotzinapa no estaban laborando. Empezarían actividades pues habían perdido la lucha y no había otra alternativa que aceptar la reforma.

Los dirigentes estudiantiles opinaron que si en realidad existiera el propósito gubernamental de ampliar, democratizar y mejorar el sistema educativo no se incurriría en el daño que se causaba en la desorganización del sistema de escuelas normales rurales, como se aprobó. ${ }^{25}$ No obstante, los estudiantes normalistas rurales salieron derrotados, $y$ varios líderes estudiantiles, tras el fracaso de su lucha, pasarían a sumar las filas de la guerrilla.

\section{Conclusiones}

Las normales rurales fueron un proyecto educativo producto de la Revolución de I9IO, cuyas demandas campesinas eran justicia social y el reparto agrario. Las normales rurales, desde su creación, sufrieron muchas reformas, entre ellas las de 1934, I94I y 1969. Los estudiantes normalistas rurales recibieron una formación política

25 "La lucha contra la agresión a las normales rurales", en $\mathrm{La}$ Voz de México, 6 de octubre de 1969, p. 6 . 
en los órganos de gobierno escolar, en la sociedad de alumnos y los comités de participación política e ideológica. Pero no todos los estudiantes simpatizaron con los movimientos sociales y estudiantiles; unas escuelas tuvieron más participación activa que otras.

Las ENR ubicadas en el medio rural se solidarizaron con los movimientos agrarios. Las razones que condujeron a los alumnos a participar como activistas fueron el conocimiento de las condiciones de pobreza y de injusticia social de sus comunidades. La guerrilla surgió en el norte del país pero se radicalizó en el sur, por las condiciones más difíciles.

La participación activa de algunos líderes y de algunas escuelas en estos movimientos fue un capítulo oscuro que manchó el nombre y la función de dichas instituciones. La reforma de 1969 fue acertada al separar el nivel de secundaria del nivel de normal, y lograr exigir el requisito obligatorio de estudios secundarios previos, y un paso importante en la profesionalización de la carrera de maestro. Pero no era necesario quitar o disminuir el número de normales rurales, sino crear nuevas secundarias.

Es importante reflexionar sobre cómo se fue articulando el sistema educativo y sobre la utilidad de la educación rural para las necesidades sociales. En 1969 no solo se reformaron las normales rurales sino también se intentó reorganizar el subsistema de educación rural, al diseñar un proyecto de creación de nuevas instituciones de enseñanza media y superior para la población del campo. La preocupación por la educación rural llevó a crear las ETA, luego los Centros de Estudios Tecnológicos Agropecuarios (CETA), que fueron concebidos como carreras cortas, pero los estudiantes las utilizaron como trampolín para que fueran reconocidos sus estudios de bachillerato técnico y de ese modo continuar con estudios universitarios. Son el antecedente de los Centros de Bachillerato Tecnológico Agropecuario (свтAs). Un modelo de educación rural (educación media superior) que fue exitoso pero hoy se encuentra deteriorado.

Este texto es una aproximación al movimiento estudiantil en el normalismo rural y su participación en el 68. Es necesario esclarecer sus momentos difíciles y comprender la situación y los problemas actuales de las normales rurales para replantear su presente y su futuro. La reforma educativa del 2013 le quitó funcionalidad a las escuelas normales. Es necesario devolverles su papel histórico como formadoras de maestros. El panorama para dichas instituciones no ha mejorado, siguen sufriendo agresiones, continúan en el abandono con precariedades. El movimiento de los estudiantes normalistas rurales pugnó por conservar el modelo de educación rural, 
que en la actualidad muestra bajas matriculas, por el desprestigio y el olvido, y su futuro es incierto.

El proceso social y político de ese momento histórico de 1968 implicó un antes y un después para las ENR. El fin de una etapa y el comienzo de otra. Las ENR continuaron siendo afectadas. Las ENR después de 1968 pasaron un periodo difícil en los años setenta y ochenta, que es un tema a seguir en otro momento.

\section{Fuentes:}

Archivo General de la Nación (AGN)

Fondos: Secretaría de Gobernación (sG)

Secciones:

+ Dirección Federal de Seguridad (DFs)

+ Investigaciones Políticas y Sociales (IPs).

\section{Referencias}

Civera, Cerecedo, Alicia

2008 La escuela como opción de vida. La formación de maestros normalistas rurales en México (1921-1945), Zinacantepec, Estado de México, El Colegio Mexiquense.

Castellanos, Laura 2007 México armado 1943-1981, México, Ediciones Era.

Cuevas, J. Aurelio

I984 El Partido Comunista Mexicano, 1963-1973, México, Universidad Autónoma de Guerrero/Universidad Autónoma de Zacatecas, Editorial Línea, Serie Estado y Educación en México.

Del Castillo Troncoso, Alberto

2012 Ensayo sobre el movimiento estudiantil de 1968. La fotografía y la construcción de un imaginario, México, Instituto Mora, IISUE-UnAm.

Flores Méndez Yessenia

2018 "Nosotros tenemos identidad de maestros y corazón de labriegos" Identidad y resistencia en la Normal Rural de Tamatán, Tamaulipas, 1930-1969, 
tesis de doctorado en Ciencias Sociales, El Colegio de San Luis, A.C, México.

García Aguirre, Aleida

2012 Normalistas y maestros en el movimiento campesino y guerrillero de Chibuabua, 1960-1968, tesis de Maestría en Ciencias con especialidad en Investigación Educativa, Die-Cinvestav, México.

García Aguirre, Aleida

2015 La Revolución que llegaría. Experiencias de solidaridad y redes de maestros y normalistas en el movimiento campesino y la guerrilla moderna en Chibuabua, 1960-1968, México, Colectivo Memorias Subalternas.

Guevara Niebla, Gilberto

1988 La democracia en la calle. Crónica del movimiento estudiantil mexicano, México, Siglo xxi Editores/Instituto de Investigaciones Sociales-unAm.

Greaves, Cecilia

2008 De radicalismo a la unidad nacional. Una visión de la educación en el México Contemporáneo (1940-1964), México, El Colegio de México.

Hernández Santos, Marcelo

2013 En tiempos de la reforma: Politicas y configuración de las reformas educativas en la Escuela Normal Rural de San Marcos, Zacatecas, 1926-1984, tesis de Doctorado en Historia, Universidad Autónoma de Zacatecas, México.

López Macedonio, Mónica Naymich,

2016 Historia de una relación institucional. Los estudiantes normalistas rurales organizados en la Federación de Estudiantes Campesinos Socialistas de México y el Estado mexicano del siglo Xx, 1935-1969, tesis de Doctorado en Historia, El Colegio de México, México.

Meyer, Lorenzo

2000 "De la estabilidad al cambio" en Historia General de México, El Colegio de México, México.

Scott, James

2000 Los dominados y el arte de la resistencia, Discursos ocultos, México, Era.

Ortiz Briano, Sergio

20II Movimiento estudiantil en el normalismo rural mexicano. Del cardenismo a la apertura democrática. Una mirada desde Cañada Honda, Aguascalientes, 1920-1979, tesis de Doctorado en Historia, Universidad Autónoma de Zacatecas, Zacatecas.

Oikión Verónica y Marta Eugenia García Ugarte (eds.) 
2008 Movimientos armados en México, siglo Xx, volumen I, México, El Colegio de Michoacán/ciesas.

Ortiz Briano Sergio y Salvador Camacho

2017 "El normalismo rural mexicano y la 'conjura comunista' de los años sesenta. La experiencia estudiantil de Cañada Honda, Aguascalientes", Revista Mexicana de Historia de la Educación, 5(Iо), pp. 245-266.

Partido Comunista Mexicano (PCM)

1973 Partido Comunista Mexicano, 1967-1972, México, Ediciones de Cultura Popular.

Peláez, Gerardo

1980 El Partido Comunista Mexicano, 60 años de historia (Cronología 19191968), Culiacán, Universidad Autónoma de Sinaloa.

Zermeño, Sergio

1978 México: una democracia utópica. El movimiento estudiantil de 1968, México, Siglo xxi Editores.

Yessenia Flores Méndez

Licenciada en Ciencias de la Educación por la Universidad Autónoma de Tamaulipas, maestra en Historia por la Universidad de Guanajuato y doctora en Ciencias Sociales por el Colegio de San Luis. Su línea de investigación es historia de la educación. Actualmente es investigadora de El Colegio de Tamaulipas. Correo: yessenia.flores@tam.gob.mx

Citar como: Yessenia Flores Méndez (2019),"Escuelas Normales Rurales en México: movimiento estudiantil y guerrilla", Iztapalapa. Revista de Ciencias Sociales y Humanidades, núm. 87, año 40, julio-diciembre de 2019, ISSN: 2007-9176; pp. 205-226. Disponible en <http://revistaiztapalapa.izt.uam. $\mathrm{mx} /$ index.php/izt/issue/archive $>$. 